\title{
Spreading of complex regional pain syndrome: not a random process
}

\author{
Monique A. van Rijn · Johan Marinus • \\ Hein Putter • Sarah R. J. Bosselaar • \\ G. Lorimer Moseley $\cdot$ Jacobus J. van Hilten
}

Received: 22 December 2010/ Accepted: 6 February 2011/Published online: 18 February 2011

(C) The Author(s) 2011. This article is published with open access at Springerlink.com

\begin{abstract}
Complex regional pain syndrome (CRPS) generally remains restricted to one limb but occasionally may spread to other limbs. Knowledge of the spreading pattern of CRPS may lead to hypotheses about underlying mechanisms but to date little is known about this process. The objective is to study patterns of spread of CRPS from a first to a second limb and the factors associated with this process. One hundred and eighty-five CRPS patients were retrospectively evaluated. Cox's proportional hazards model was used to evaluate factors that influenced spread of CRPS symptoms. Eighty-nine patients exhibited CRPS in multiple limbs. In 72 patients spread from a first to a second limb occurred showing a contralateral pattern in $49 \%$, ipsilateral pattern in $30 \%$ and diagonal pattern in $14 \%$. A trauma preceded the onset in the second limb in 37, 44 and $91 \%$, respectively. The hazard of spread of CRPS increased with the number of limbs affected. Compared to patients with CRPS in one limb, patients with CRPS in multiple limbs were on average 7 years younger and more often had movement disorders. In patients with CRPS in multiple limbs, spontaneous spread of symptoms generally follows a contralateral or ipsilateral pattern whereas
\end{abstract}

M. A. van Rijn $(\bowtie) \cdot J$. Marinus · S. R. J. Bosselaar ·

J. J. van Hilten

Department of Neurology (K5Q), Leiden University Medical

Center, P.O. Box 9600, 2300 RC Leiden, The Netherlands

e-mail: m.a.van_rijn@lumc.nl

H. Putter

Department of Medical Statistics, Leiden University Medical Center, Leiden, The Netherlands

G. L. Moseley

Prince of Wales Medical Research Institute \& The University of New South Wales, Corner of Easy \& Barker Streets,

Randwick 2031, Australia diagonal spread is rare and generally preceded by a new trauma. Spread is associated with a younger age at onset and a more severely affected phenotype. We argue that processes in the spinal cord as well as supraspinal changes are responsible for spontaneous spread in CRPS.

Keywords CRPS $\cdot$ Spread $\cdot$ Multiple $\cdot$ Peripheral trauma $\cdot$ TREND Study

\section{Introduction}

Complex regional pain syndrome (CRPS) is characterized by various combinations of sensory, autonomic and motor disturbances, and is usually preceded by a minor to severe trauma affecting a limb (Allen et al. 1999; Merskey and Bogduk 1994; Veldman et al. 1993). CRPS usually remains restricted to one limb, but it can spread to other body parts (Maleki et al. 2000; Veldman and Goris 1996). Although several small studies have reported spread of specific sensory, autonomic or motor features of the syndrome, the overall picture remains unclear (Bhatia et al. 1993; Maleki et al. 2000; van Hilten et al. 2001; Veldman and Goris 1996). CRPS in one limb may extend to another limb either as a result of a new trauma to a previously unaffected limb, or because the syndrome spreads spontaneously. Although different causes of spontaneous spread have been proposed, including genetic predisposition, aberrant regulation of neurogenic inflammation and maladaptive neuronal plasticity, the underlying mechanisms have not been elucidated (Maleki et al. 2000; van Rijn et al. 2007; Veldman and Goris 1996).

As a tertiary care center for CRPS we were able to characterize a large sample of patients in whom CRPS in one limb spread into involve another limb. We were 
particularly interested in patients in whom spreading occurred spontaneously (i.e., without a new trauma), because this may reflect true spread of the disorder, which might provide important information about the mechanisms behind this process. For example, if systemic factors underpin spontaneous spread, then one would expect an indiscriminate pattern of spread; if cortical mechanisms underpin spontaneous spread, then one would expect an ipsilateral pattern, and if spinal mechanisms underpin spontaneous spread, then one would expect a contralateral pattern.

The present study aims to evaluate patterns of spread of CRPS from one to a second limb and consider potential mechanisms that could explain this process. In addition, factors that are associated with the occurrence of spread are studied.

\section{Methods}

\section{Patients}

All patients who visited the outpatient movement disorders clinic of the Department of Neurology of the Leiden University Medical Center from January 1998 to April 2004 were considered for inclusion in the study. Patients were eligible if they met the CRPS criteria of the International Association for the Study of Pain (IASP), either at the time of disease onset or at the time of presentation at the clinic. The IASP criteria include the combination of: (1) the presence of an initiating noxious event or a cause of immobilization, (2) continuing pain, allodynia or hyperalgesia with which the pain is disproportionate to any inciting event, (3) evidence at some time of edema, changes in skin blood flow or abnormal sudomotor activity in the region of the pain and (4) absence of a condition that would otherwise account for the degree of pain and dysfunction. Although only criteria 2-4 have to be satisfied (Merskey and Bogduk 1994), we only included patients who identified an initiating noxious event in the first affected limb.

\section{Data collection}

Dates of onset of CRPS signs or symptoms for every involved limb were obtained from the patient's history. Medical records were reviewed to verify data wherever possible. Sensory features that were recorded included pain, hypoalgesia, hyperalgesia and allodynia. Hypoalgesia, hyperalgesia and allodynia were assessed by testing sensitivity to light touch and pinprick. Recorded autonomic features involved oedema, temperature changes, colour changes, hyper- or hypohidrosis and changes in nail and hair growth. Recorded movement disorders included dystonia, tremor and myoclonus. We did not consider muscle weakness a movement disorder as this could result from pain or oedema. For all affected limbs we evaluated if the symptoms and signs fulfilled the IASP criteria for CRPS. Age at onset in the first limb and length of interval to onset of symptoms in subsequent limbs were calculated. The presence and type of traumas (soft tissue injury, fracture, surgery) preceding CRPS were registered. We categorized patients according to three criteria. First, if CRPS was present in one limb, patients were categorized as 'Single-CRPS'. If CRPS was present in more than one limb, they were categorized as 'Multiple-CRPS'. Second, Multiple-CRPS cases were categorized according to whether spread was associated with a separate trauma to the limb. If not, patients were categorized as 'Spontaneous spread'. If so, they were categorized as 'Separate trauma'. Third, Multiple-CRPS cases were categorized according to which limb was subsequently affected: 'Contralateral' (e.g. left hand to right hand), 'Ipsilateral' (e.g. left hand to left leg) or 'Diagonal' (e.g. left hand to right leg).

\section{Statistical analysis}

The independent-samples $t$ test was used to assess differences between groups in normally distributed continuous data, while non-parametric tests were used to assess differences in non-normally distributed continuous or categorical data. Baseline differences in disease duration were taken into account and analyzed with analysis of covariance. The time from onset of initial symptoms to extension to other limbs was calculated for each limb, where time to spread was censored at the time of last assessment. In patients who showed spontaneous spread of symptoms to subsequent limbs, a multivariate analysis of factors associated with spread of symptoms was carried out with Cox's proportional hazards model. This analysis involves a regression model to quantify the relationships between one or more factors of interest and 'survival' (time to the occurrence of spread). At any point in time, an individual has an instantaneous risk ("hazard") to reach the endpoint (in our study defined as "spread to a second limb"). The hazard ratio presents the increased or decreased risk on reaching the endpoint at any point in time (compared to a reference value), adjusted for other potentially confounding variables in the model. Patients with a simultaneous onset of symptoms in more than one limb or with simultaneous spread from one affected limb to more than one subsequent limb were excluded from this analysis. The hazard of spread was estimated while several variables were accounted for, including trauma characteristics, location of initial symptoms, presence of movement disorders and patient characteristics. The probabilities of spread to other limbs were calculated as cumulative incidences (competing 
risks) (Putter et al. 2007). For the analysis of rate of spread comparing the presence of one, two or three affected limbs, the variance of the estimated coefficients was adjusted using a sandwich estimator, accounting for possible correlations of event times within patients (Lin and Wei 2009). $P$ values $\leq 0.05$ were considered significant. All statistical analyses were performed with SPSS (version 14.0), except for the survival analyses, which were performed with the statistical program $\mathrm{R}$ (version 2.0.1).

\section{Results}

One-hundred and eighty-five patients were included in the study (Table 1, Fig. 1). During assessment, 96 patients $(52 \%)$ had a single affected limb, whereas $89(48 \%)$ had multiple affected limbs. The signs and symptoms are presented in Table 2. In the Multiple-CRPS group, the syndrome started in 1 limb in 78 patients (i.e. 88\%), a simultaneous start in 2 limbs in 10 patients (11\%) and a simultaneous start in 4 limbs in 1 patient (1\%).

Spread of CRPS from one to two limbs

CRPS had spread to another limb in 78 patients. Spread occurred simultaneously from one to three limbs in five patients and from one to four limbs in one patient (Fig. 1, section A). The severity of CRPS symptoms in the second

Table 1 Demographics of 185 patients with CRPS

\begin{tabular}{lc}
\hline Characteristic & Value \\
\hline Females: no. (\%) & $160(86.5)$ \\
Disease duration, mean (SD) (years) & $6.0(6.0)$ \\
Age at assessment, mean (SD) (years) & $43.5(14.8)$ \\
Age at onset of CRPS, mean (SD) (years) & $37.5(15.4)$ \\
Preceding trauma, no. (\%) & \\
Soft tissue injury & $92(49.7)$ \\
Fracture & $48(25.9)$ \\
Surgery & $45(24.3)$ \\
CRPS involvement, no. (\%) & \\
Single limb & $96(51.9)$ \\
Multiple limbs & $89(48.1)$ \\
Affected limbs at initial CRPS onset, no. (\%) & \\
1 & $78(87.6)$ \\
2 & $10(11.2)$ \\
3 & 0 \\
4 & $1(1.1)$ \\
Affected limbs at assessment, no. (\%) & \\
2 & $45(50.6)$ \\
3 & $18(20.2)$ \\
4 & $26(29.2)$ \\
\hline
\end{tabular}

limb did not differ significantly from that in the first limb (Table 2). CRPS spread from 1 to 2 limbs in 72 patients according to the following patterns (Table 3 ): contralateral pattern in 38 patients $(53 \% ; 22$ arm to arm, 16 leg to leg); ipsilateral pattern in 23 patients $(32 \% ; 12$ arm to leg, 11 leg to arm) and diagonal pattern in 11 patients (15\%). New trauma preceded the onset of CRPS in the second limb in $37 \%$ of the patients with contralateral spread, in $44 \%$ of the patients with ipsilateral spread and in $91 \%$ of the patients with diagonal spread, which indicates that diagonal spreading is almost always associated with a new trauma. Patient characteristics did not differ between the three types of spread.

\section{Spontaneous spread versus spread after separate trauma}

In 38 patients who showed spontaneous spread of CRPS from a first to a second limb, contralateral spread occurred in 24 patients $(63 \%, 11$ arm to arm and 13 leg to leg) (Table 3). Ipsilateral spread occurred in 13 (34\%, 8 arm to leg and 5 leg to arm) and diagonal spread occurred in 1 patient (3\%). In 34 patients who showed spread after a separate trauma of the second limb, contralateral spread occurred in 14 (41\%, 11 arm to arm and 3 leg to leg) patients. Ipsilateral spread occurred in $10(29 \%, 4$ arm to leg and 6 leg to arm) and diagonal spread occurred in 10 (29\%, 4 arm to leg and 6 leg to arm). Patterns of spread were significantly different between patients with spontaneous spread and spread after a separate trauma $\left(\chi^{2}(2)=10.2 ; P=0.006\right)$. Patient characteristics (including symptom severity) did not differ significantly between patients who spread spontaneously and those who spread after a separate trauma. Patients in whom spreading occurred spontaneously showed a non-random pattern of spread, so further analyses were performed on data from this subgroup.

\section{Characteristics of spontaneous spread}

The median interval between the occurrence in the first and second limb was 21 months $(n=24$, range $2-95)$ for contralateral spread, 19 months $(n=13$, range 3-58) for ipsilateral spread and 10 months $(n=1)$ for diagonal spread. The difference in intervals between contralateral and ipsilateral pattern was not significant (Mann-Whitney $U$ test; $P=0.16$ ).

Next, the hazard of the different types of spontaneous spread was calculated (Table 4). Compared to patients with contralateral pattern (reference value of 1.00) the hazard of ipsilateral spread was 0.44 (95\% CI: $0.22-0.89)$, whereas the hazard of diagonal spread was 0.04 (CI 0.005-0.30) (Fig. 2). Age at onset, sex, or onset of symptoms in arm or 
Fig. 1 Flow diagram of patients included in the study. Section A shows the included patients in which CRPS symptoms spread from one to two limbs and who were evaluated for different patterns of spread. Section B shows the included patients with multiple and single affected limbs that were compared for differences in clinical characteristics

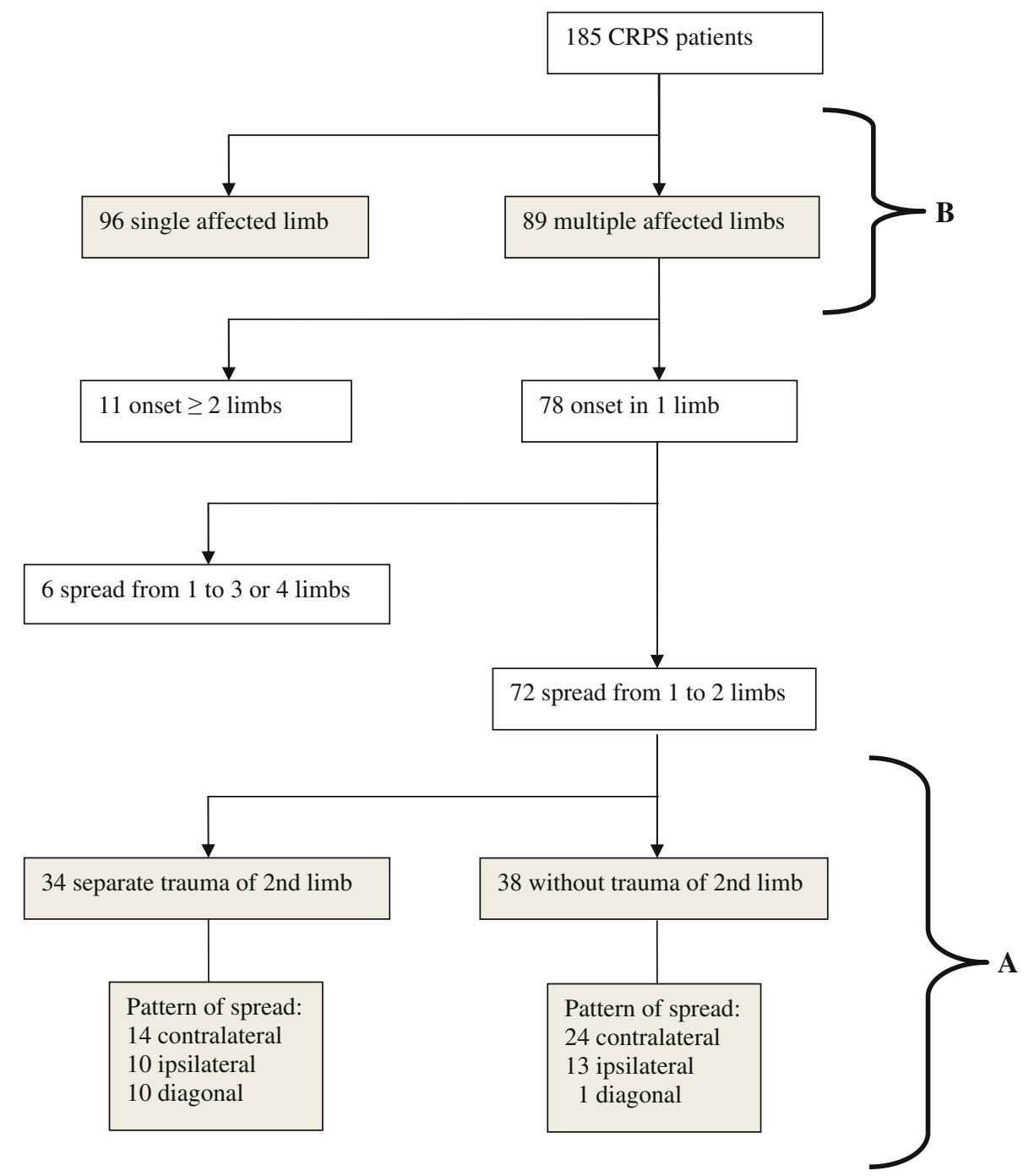

leg, or in left or right sided limbs, did not affect the hazard. Compared to the presence of CRPS in one limb, the presence in two limbs increased the hazard of spread of CRPS to a third limb with 2.19 (95\% CI: 1.35-3.57). CRPS in three limbs increased the hazard of spread to a fourth limb to 3.75 (95\% CI: 1.92-7.32). The hazard of spread in patients with onset of CRPS on the left side was 1.46 (95\% CI: $1.00-2.11, P=0.047)$ compared to patients with rightsided onset, indicating a somewhat higher risk of spread of CRPS symptoms in patients with left sided onset.

\section{Comparison of Single- and Multiple-CRPS patients}

Ninety-six patients with Single-CRPS were compared with 89 patients with Multiple-CRPS (Fig. 1, section B). Patients with Multiple-CRPS had longer disease duration, and were significantly younger at onset, than patients with Single-CRPS (Table 5). Additional analyses with adjustment for differences in disease duration showed that patients with Multiple-CRPS were 6.7 years younger (95\% CI: 6.3-7.1). There was no significant difference in the type of trauma $\left(\chi^{2}(2)=5.67 ; P=0.06\right)$ between groups. Movement disorder was more common in those with Multiple-CRPS than it was in those with Single-CRPS $[78 \%$ vs. $54 \%$, mean $(95 \% \quad \mathrm{CI})$ difference $=23 \%$ $(10-37 \%)]$. No difference between the groups was found in the type of sensory symptoms $\left(\chi^{2}(2)=0.73 ; P=0.69\right)$. Patients with spontaneous spread had a shorter disease duration than those with secondary trauma-related spread (6.4 vs. 9.6 years, mean difference 3.2 years, $95 \%$ CI: $0.4-5.8$ ) but there were no other differences between these two groups.

\section{Discussion}

We set out to determine patterns of spread of CRPS and the factors that are associated with spread. Our results show 
Table 2 Signs and symptoms of CRPS in affected limbs

\begin{tabular}{|c|c|c|c|c|}
\hline \multirow[t]{2}{*}{ Variable } & \multicolumn{4}{|l|}{ Affected limb } \\
\hline & First $(n=185)$ & Second $(n=89)$ & Third $(n=44)$ & Fourth $(n=26)$ \\
\hline \multicolumn{5}{|l|}{ Pain } \\
\hline Present/absent/unknown, no. & $185 / 0 / 0$ & $89 / 0 / 0$ & $44 / 0 / 0$ & $26 / 0 / 0$ \\
\hline \multicolumn{5}{|l|}{ Hyperalgesia/allodynia } \\
\hline Present/absent/unknown, no. & $101 / 78 / 6$ & $40 / 48 / 1$ & $19 / 24 / 1$ & $10 / 16 / 0$ \\
\hline \multicolumn{5}{|l|}{ Hypoalgesia } \\
\hline Present/absent/unknown, no. & $152 / 30 / 3$ & $72 / 17 / 0$ & $39 / 5 / 0$ & $23 / 3 / 0$ \\
\hline \multicolumn{5}{|l|}{ Edema } \\
\hline Present/absent/unknown, no. & $168 / 9 / 8$ & $67 / 20 / 2$ & $27 / 13 / 4$ & $17 / 8 / 1$ \\
\hline \multicolumn{5}{|l|}{ Temperature changes } \\
\hline Present/absent/unknown, no. & $165 / 9 / 11$ & $73 / 9 / 7$ & $41 / 2 / 1$ & $21 / 4 / 1$ \\
\hline \multicolumn{5}{|l|}{ Color changes } \\
\hline Present/absent/unknown, no. & $176 / 3 / 6$ & $82 / 5 / 2$ & $33 / 7 / 4$ & $24 / 2 / 0$ \\
\hline \multicolumn{5}{|l|}{ Hyper/hypohidrosis } \\
\hline Present/absent/unknown, no. & $122 / 44 / 19$ & $59 / 27 / 3$ & $26 / 15 / 3$ & $13 / 12 / 1$ \\
\hline \multicolumn{5}{|l|}{ Hair and nail growth changes } \\
\hline Present/absent/unknown, no. & $134 / 42 / 9 /$ & $52 / 32 / 5$ & $27 / 13 / 4$ & $18 / 7 / 1$ \\
\hline \multicolumn{5}{|l|}{ Movement disorders ${ }^{\mathrm{a}}$} \\
\hline Present/absent/unknown, no. & $115 / 70 / 0$ & $67 / 22 / 0$ & $36 / 8 / 0$ & $25 / 1 / 0$ \\
\hline
\end{tabular}

Variables were deemed to be present if a symptom, a sign or both were reported or observed

${ }^{a}$ Recorded movement disorders were dystonia, tremor and myoclonus

Table 3 Patterns of spread in 72 patients who spread from one to two limbs spontaneously or after a separate trauma of the second extremity

\begin{tabular}{llll}
\hline Pattern of spread $^{\text {a }}$ & Total $(N=72)$ & Spontaneous spread $(N=38)$ & Separate trauma $(N=34)$ \\
\hline Contralateral, no. $(\%)$ & $38(53)$ & $24(63)$ & $14(41)$ \\
Ipsilateral, no. $(\%)$ & $23(32)$ & $13(34)$ & $10(29)$ \\
Diagonal, no. $(\%)$ & $11(15)$ & $1(3)$ & $10(29)$
\end{tabular}

${ }^{a}$ Patterns of spread were significantly different between patients with spontaneous spread and spread after a separate trauma; $\chi^{2}(2)=10.2$; $P=0.006$

that CRPS usually affects one limb but in some cases it spreads to another limb, most often in a contralateral (53\%) or ipsilateral $(32 \%)$ pattern and usually without secondary trauma. A diagonal pattern of spread was nearly always triggered by a new trauma. Spontaneous spread and spread after a separate trauma followed different patterns.

The mechanism underlying spontaneous spread of CRPS to other limbs is unclear. Common patterns of spontaneous spread of CRPS may hint at the origin of the pattern. Spread after a separate trauma followed no particular pattern, which strongly suggests that CRPS in one limb does not specifically predispose a particular other limb to CRPS and supports the idea that these patients have multiple CRPS rather than CRPS of multiple limbs. In contrast, spontaneous spread to the contralateral limb was 2.3 times more likely that spread to the ipsilateral limb and 25 times more likely than diagonal spread. This result casts light on previous reports of similar rates of ipsilateral and diagonal spread (Veldman and Goris 1996) because that work did not differentiate between spontaneous and second traumarelated spread.

Patients with a spontaneous onset or who have a familial form of CRPS develop the syndrome at a younger age and are more likely to have a more severe phenotype (de Rooij et al. 2009a). Additionally, CRPS patients younger than 50 have an increased risk of having siblings with CRPS (de Rooij et al. 2009b). In line with these studies, patients with Multiple-CRPS more often exhibited movement disorders and also had a significantly younger age at onset of CRPS than patients with Single-CRPS. Collectively, these findings indicate that in patients with a younger onset of CRPS, genetic factors may play a role in the onset or 
Table 4 Hazard on spread of CRPS-Multivariate Cox regression model

\begin{tabular}{lcc}
\hline Variable & Hazard ratio & $95 \%$ CI \\
\hline Pattern of spread to second affected limb & \\
Mirror-image & 1 & \\
Ipsilateral & 0.44 & $0.22-0.89$ \\
Diagonal & 0.04 & $0.005-0.30$ \\
Onset in limb & & \\
Right sided & 1 & \\
Left sided & 1.46 & \\
Number of limbs already affected by CRPS & \\
1 & 1 & $1.00-2.11$ \\
2 & 2.19 & $1.92-7.32$ \\
3 & 3.75 & \\
\hline
\end{tabular}

${ }^{a}$ Regression coefficient with $95 \%$ CI

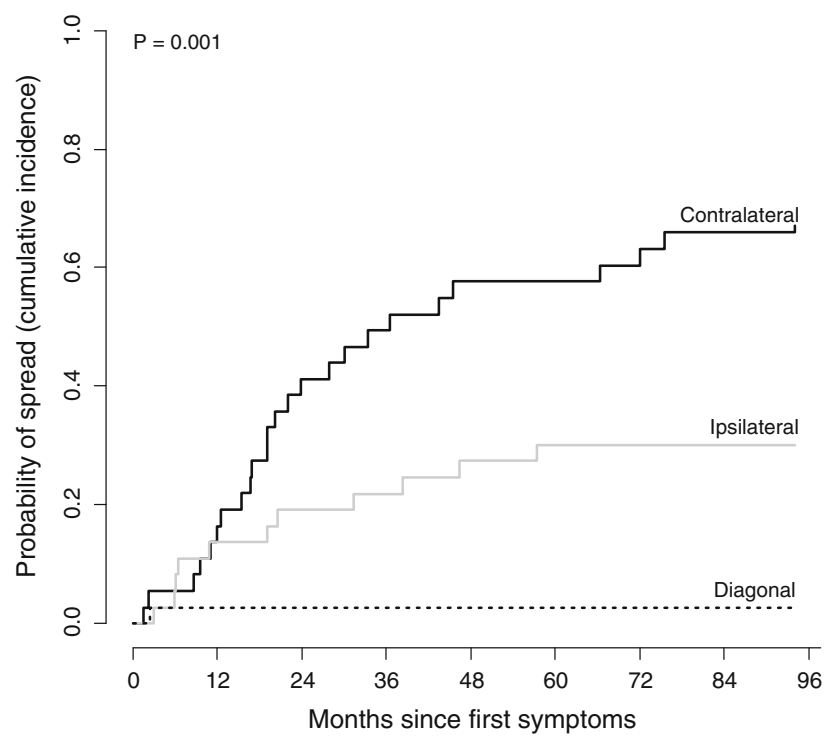

Fig. 2 The probability of spread of CRPS. The probability on the occurrence of different types of spread in CRPS patients since the onset of symptoms in the first limb. In this multivariate model differences in patient characteristics were accounted for

chronicity of the syndrome. A genetic predisposition is also suggested by associations that were found with different human leukocyte antigen (HLA) class I and II factors (de Rooij et al. 2009c; Kemler et al. 1999; van Hilten et al. 2000; Vaneker et al. 2002). Interestingly, HLA class I molecules have been implicated in non-immune roles including neuroplasticity (Corriveau et al. 1998; Goddard et al. 2007).

The dominant patterns of spontaneous spread observed here strongly suggest that CRPS does not spread according to some systemic vulnerability, but is more likely to spread via spinal or cortically mediated mechanisms.
Pain that spreads contralaterally has been reported in CRPS and other chronic pain conditions, such as atypical facial pain (Woda and Pionchon 2000), phantom limb pain (Pohjolainen 1991) and repetitive strain injury (Miller and Topliss 1988). Several animal models of neuropathic pain and CRPS have reported contralateral spread of symptoms after nerve lesions or inflammation (Coderre et al. 2004; Coderre and Melzack 1992; Koltzenburg et al. 1999). In a recent rat model of CRPS, $57 \%$ of the animals exhibited contralateral hindpaw mechanical hypersensitivity after unilateral needle stick distal nerve injury (Siegel et al. 2007). Following an intradermal injection of capsaicin, human subjects developed contralateral hyperalgesia and allodynia (Shenker et al. 2008). The etiology behind the contralateral spread of pain is largely unknown; however, increasing evidence from experimental studies on neuropathic pain suggests that contralateral changes arise via altered spinal processing of incoming sensory information (Koltzenburg et al. 1999; Watkins and Maier 2002). This may be mediated by growth factors via commissural interneurons in the spinal cord and brainstem. In addition, spinal glia cells and pro-inflammatory cytokines have been documented as important factors behind the contralateral spread of symptoms (Hatashita et al. 2008; Milligan et al. 2003).

In contrast to the number of studies on contralateral spread, data on mechanisms underlying spread of symptoms to the ipsilateral limb are scarce. Axial spread of disease along the spinal cord is well documented for degenerative diseases such as amyotrophic lateral sclerosis and infectious agents such as the poliovirus (Brooks 1991). It is conceivable that glial mediated changes at one segment of the spinal cord can reach remote segments by axonal transport via descending or ascending fibre tracts. This is also suggested by a recent autopsy paper on a patient with longstanding CRPS that started in the left leg, but eventually spread to all limbs (Del Valle et al. 2009). The researchers demonstrated a significant loss of posterior horn cells and activation of both microglia and astrocytes not only at the site of the initial injury, but also extending throughout the entire length of the spinal cord. These diffuse alterations may support the hypothesis that segmental changes in the spinal cord induced by CRPS in one limb, may not only spread to the contralateral side but can also extend more rostrally and caudally from the initially affected segment. Interestingly, this latter study (Del Valle et al. 2009) also reported that the greatest degree of microglial cell activation in the spinal cord was seen in the left lumbar segments and the least in the right cervical cord, which suggests that ispilateral changes are induced more easily than diagonal changes.

Another explanation for the spread of symptoms may be found at the supraspinal level. Rommel et al. (1999) 
Table 5 Comparison of characteristics of CRPS patients with single and multiple affected limbs

\begin{tabular}{|c|c|c|c|c|}
\hline Parameter & Total $(N=185)$ & Single $(N=96)$ & Multiple $(N=89)$ & Difference in $\%(95 \% \mathrm{CI})$ \\
\hline Female-no. $(\%)$ & $160(86.5)$ & $84(87.5)$ & $76(85.4)$ & $2.1(-10.6 ; 14.8)$ \\
\hline First aff. limb arm-no. (\%) $n=174$ & $91(52.3)$ & $50(52.1)$ & $41(52.6)$ & $0.5(-14.4 ; 15.4)$ \\
\hline Disease duration-mean (SD) yr & $6.0(6.0)$ & $4.1(4.7 \mathrm{t})$ & $8.1(6.6)$ & $4.0(2.3-.7)$ \\
\hline Age at onset CRPS-mean (SD) year & $37.5(15.4)$ & $40.7(14.7)$ & $34.0(14.7)$ & $6.7(6.3 ; 7.1)$ \\
\hline \multicolumn{5}{|l|}{ Kind of trauma-no. $(\%)$} \\
\hline Soft tissue injury & $92(49.7)$ & $43(44.8)$ & $49(55.1)$ & \multirow{3}{*}{$\begin{array}{l}\chi^{2}(d f=2) \\
P=0.06\end{array}$} \\
\hline Fracture limb/other & $48(25.9)$ & $32(33.3)$ & $16(18.0)$ & \\
\hline Operation limb/other & $45(24.3)$ & $21(21.9)$ & $24(27.0)$ & \\
\hline Movement disorders-no. (\%) & $121(65.4)$ & $52(54.2)$ & $69(77.5)$ & $23.3(10.1 ; 36.5)^{\mathrm{a}}$ \\
\hline \multicolumn{5}{|l|}{ Type sensory symptoms-no. (\%) $n=165$} \\
\hline Hypaesthesia/hypalgesia & $81(49.1)$ & $43(52.4)$ & $38(45.8)$ & \multirow{3}{*}{$\begin{array}{l}\chi^{2}(d f=2)=0.73 \\
P=0.69\end{array}$} \\
\hline Hyperaesthesia/hyperalgesia/allodynia & $41(24.8)$ & $19(23.2)$ & $22(26.5)$ & \\
\hline Both & $43(26.1)$ & $20(24.4)$ & $23(27.7)$ & \\
\hline
\end{tabular}

${ }^{\text {a }}$ Adjusted for disease duration

showed hemisensory impairment in CRPS patients with only one affected limb, and that this was more common in those with left-sided CRPS. They proposed that the result may reflect functional alterations in the thalamus. Relevant to this is the recent discovery of space-based, but not armbased shift in tactile processing in people with CRPS of one arm (Moseley et al. 2009). Of further relevance here is the observation that left-sided CRPS is associated with a higher hazard of spontaneous spread-space-based tactile neglect after stroke commonly involves the left side of the body, consequent to right sided brain damage (Bisiach et al. 1979).

Contralateral spread probably involves different supraspinal mechanisms. Noxious stimuli activate bilateral regions of the brain associated with descending control pathways including the thalamus and rostral ventral medulla, which suggests one putative mechanism for mediating altered spinal gating contralaterally (Bantick et al. 2002; Urban and Gebhart 1999). Additionally, the growing body of data implicating cortical changes in CRPS (see Swart et al. 2009 for review), offer potential mechanisms. For example, watching the mirror-image of the unaffected limb being touched elicits pain on the affected side (Acerra and Moseley 2005) and referred sensations of a tactile or painful stimulus were also experienced outside its expected somatic territory (McCabe et al. 2003). Forrs et al. (2005) describe a patient with chronic CRPS type- 1 in whom pain and motor symptoms spread to the contralateral arm, and whole-head-magnetoencephaloghy demonstrated abnormal bilateral activation in the primary somatosensory cortices to unilateral tactile stimuli, which suggests that interhemispheric spread of cortical activation may contribute to contralateral spread. Furthermore, supraspinal glia and glial-derived proinflammatory cytokines may play a role in spread of symptoms as well as their major influence on pain modulation (Watkins and Maier 2002). Whether these supraspinal changes can initiate spread of CRPS symptoms or if they are secondary to peripheral or spinal processes remains to be elucidated.

Our study demonstrates that if CRPS develops spontaneously in more than one limb, there is a greater risk of spread to subsequent limbs without the requirement of a new trauma. This accelerated occurrence has been documented for clinical manifestations of other diseases and probably reflects changes in the central nervous system, perhaps in an attempt to adapt to the altered condition by remodelling of neuronal contacts and circuits, a process also known as neuronal plasticity (Harrison 1999; Linazasoro 2005; Sutula 2004; Woolf and Salter 2000).

Interpretation of our results should consider some methodological issues and limitations. A retrospective design is less accurate than prospective designs and may result in incomplete data, although such issues would seem unlikely to bias the results in one direction over another. Furthermore, follow-up data were not available and SingleCRPS patients had shorter disease duration than patients with Multiple-CRPS, which raises the possibility that some Single-CRPS patients would have ultimately developed Multiple-CRPS if we left it longer to find out. We addressed this issue by controlling for disease duration in the analysis. As it is likely that major traumas are better recalled than minor ones, the frequency of minor trauma may be underestimated. One can argue that these patients may be incorrectly labeled as "spontaneous spread". However, to address the objective of this study we felt it was best to use a clear definition of trauma (soft tissue 
injury, fracture, surgery) that does not include "microtraumata". Notably, this study was performed in a tertiary center for movement disorders which may lead to overrepresentation of patients with severe or multiple CRPS. However, the objective of this study was to evaluate the spread of symptoms and not the prevalence of multifocal CRPS. Finally, we are aware that this is a descriptive study, and that the pathophysiological aspects we discussed have not been tested.

In conclusion, this study shows that spread of CRPS symptoms often occurs spontaneously and contralateral spread is twice as likely as ipsilateral spread, but diagonal spread is rare. We contend that these patterns of spread implicate spinal cord and/or supraspinal mechanisms rather than systemic mechanisms, although further work is required to elucidate them in detail.

Acknowledgements This study was performed within TREND (Trauma Related Neuronal Dysfunction), a knowledge consortium that integrates research on Complex Regional Pain Syndrome type 1 and is supported by a Dutch Government Grant (BSIK03016). GLM is supported by the National Health \& Medical Research Council of Australia. The authors declare that they do not have a conflict of interest.

Open Access This article is distributed under the terms of the Creative Commons Attribution Noncommercial License which permits any noncommercial use, distribution, and reproduction in any medium, provided the original author(s) and source are credited.

\section{References}

Acerra NE, Moseley GL (2005) Dysynchiria: watching the mirror image of the unaffected limb elicits pain on the affected side. Neurology 65:751-753

Allen G, Galer BS, Schwartz L (1999) Epidemiology of complex regional pain syndrome: a retrospective chart review of 134 patients. Pain 80:539-544

Bantick SJ, Wise RG, Ploghaus A, Clare S, Smith SM, Tracey I (2002) Imaging how attention modulates pain in humans using functional MRI. Brain 125:310-319

Bhatia KP, Bhatt MH, Marsden CD (1993) The causalgia-dystonia syndrome. Brain 116(Pt 4):843-851

Bisiach E, Luzzatti C, Perani D (1979) Unilateral neglect, representational schema and consciousness. Brain 102:609-618

Brooks BR (1991) The role of axonal transport in neurodegenerative disease spread: a meta-analysis of experimental and clinical poliomyelitis compares with amyotrophic lateral sclerosis. Can J Neurol Sci 18:435-438

Coderre TJ, Melzack R (1992) The contribution of excitatory amino acids to central sensitization and persistent nociception after formalin-induced tissue injury. J Neurosci 12:3665-3670

Coderre TJ, Xanthos DN, Francis L, Bennett GJ (2004) Chronic postischemia pain (CPIP): a novel animal model of complex regional pain syndrome-Type I (CRPS-1; reflex sympathetic dystrophy) produced by prolonged hindpaw ischemia and reperfusion in the rat. Pain 112:94-105

Corriveau RA, Huh GS, Shatz CJ (1998) Regulation of class I MHC gene expression in the developing and mature CNS by neural activity. Neuron 21:505-520 de Rooij AM, de Mos M, Sturkenboom MC, Marinus J, van den Maagdenberg AM, van Hilten JJ (2009a) Familial occurrence of complex regional pain syndrome. Eur J Pain 13:171-177

de Rooij AM, de Mos M, van Hilten JJ, Sturkenboom MC, Gosso MF, van den Maagdenberg AM, Marinus J (2009b) Increased risk of complex regional pain syndrome in siblings of patients? J Pain 10:1250-1255

de Rooij AM, Florencia GM, Haasnoot GW, Marinus J, Verduijn W, Claas FH, van den Maagdenberg AM, van Hilten JJ (2009c) HLA-B62 and HLA-DQ8 are associated with complex regional pain syndrome with fixed dystonia. Pain 145:82-85

Del Valle L, Schwartzman RJ, Alexander G (2009) Spinal cord histopathological alterations in a patient with longstanding complex regional pain syndrome. Brain Behav Immun 23:8591

Forss N, Kirveskari E, Gockel M (2005) Mirror-like spread of chronic pain. Neurology 65:748-750

Goddard CA, Butts DA, Shatz CJ (2007) Regulation of CNS synapses by neuronal MHC class I. Proc Natl Acad Sci USA 104: $6828-6833$

Harrison PJ (1999) The neuropathological effects of antipsychotic drugs. Schizophr Res 40:87-99

Hatashita S, Sekiguchi M, Kobayashi H, Konno SI, Kikuchi SI (2008) Contralateral neuropathic pain and neuropathology in dorsal root ganglion and spinal cord following hemilateral nerve injury in rats. Spine 33:1344-1351

Kemler MA, van de Vusse AC, Berg-Loonen EM, Barendse GA, van Kleef M, Weber WE (1999) HLA-DQ1 associated with reflex sympathetic dystrophy. Neurology 53:1350-1351

Koltzenburg M, Wall PD, McMahon SB (1999) Does the right side know what the left is doing? Trends in Neurosci 22:122-127

Lin DY, Wei LJ (2009) Robust inference for the Cox proportional hazards model. J Am Stat Assoc 84:1074-1078

Linazasoro G (2005) New ideas on the origin of L-dopa-induced dyskinesias: age, genes and neural plasticity. Trends Pharmacol Sci 26:391-397

Maleki J, LeBel AA, Bennett GJ, Schwartzman RJ (2000) Patterns of spread in complex regional pain syndrome, type I (reflex sympathetic dystrophy). Pain 88:259-266

McCabe CS, Haigh RC, Halligan PW, Blake DR (2003) Referred sensations in patients with complex regional pain syndrome type 1. Rheumatology 42:1067-1073

Merskey H, Bogduk N (1994) Complex regional pain syndromes. In: Classification of chronic pain. Descriptions of chronic pain syndromes and definitions of pain terms.IASP Press,Seattle, pp 40-43

Miller MH, Topliss DJ (1988) Chronic upper limb pain syndrome (repetitive strain injury) in the Australian workforce: a systematic cross sectional rheumatological study of 229 patients. J Rheumatol 15:1705-1712

Milligan ED, Twining C, Chacur M, Biedenkapp J, O’Connor K, Poole S, Tracey K, Martin D, Maier SF, Watkins LR (2003) Spinal glia and proinflammatory cytokines mediate mirror-image neuropathic pain in rats. J Neurosci 23:1026-1040

Moseley GL, Gallace A, Spence C (2009) Space-based, but not armbased, shift in tactile processing in complex regional pain syndrome and its relationship to cooling of the affected limb. Brain 132:3142-3151

Pohjolainen T (1991) A clinical-evaluation of stumps in lower-limb amputees. Prosthet Orthot Int 15:178-184

Putter H, Fiocco M, Geskus RB (2007) Tutorial in biostatistics: competing risks and multi-state models. Stat Med 26:23892430

Rommel O, Gehling M, Dertwinkel R, Witscher K, Zenz M, Malin JP, Janig W (1999) Hemisensory impairment in patients with complex regional pain syndrome. Pain 80:95-101 
Shenker NG, Haigh RC, Mapp PI, Harris N, Blake DR (2008) Contralateral hyperalgesia and allodynia following intradermal capsaicin injection in man. Rheumatology 47:1417-1421

Siegel SM, Lee JW, Oaklander AL (2007) Needlestick distal nerve injury in rats models symptoms of complex regional pain syndrome. Anesth Analg 105:1820-1929

Sutula TP (2004) Mechanisms of epilepsy progression: current theories and perspectives from neuroplasticity in adulthood and development. Epilepsy Res 60:161-171

Swart CM, Stins JF, Beek PJ (2009) Cortical changes in complex regional pain syndrome (CRPS). Eur J Pain 13:902-907

Urban MO, Gebhart GF (1999) Supraspinal contributions to hyperalgesia. Proc Natl Acad Sci USA 96:7687-7692

van Hilten JJ, van de Beek WJT, Roep BO (2000) Multifocal or generalized tonic dystonia of complex regional pain syndrome: a distinct clinical entity associated with HLA-DR13. Ann Neurol 48:113-116

van Hilten JJ, van de Beek WJ, Vein AA, van Dijk JG, Middelkoop HA (2001) Clinical aspects of multifocal or generalized tonic dystonia in reflex sympathetic dystrophy. Neurology 56:17621765 van Rijn MA, Marinus J, Putter H, van Hilten JJ (2007) Onset and progression of dystonia in complex regional pain syndrome. Pain 130:287-293

Vaneker M, van de Laan L, Allebes WA, Goris JA (2002) Genetic factors associated with complex regional pain syndrome 1: HLA DRB and TNF alpha promotor gene polymorphism. Disabil Med 2:69-74

Veldman PH, Goris RJ (1996) Multiple reflex sympathetic dystrophy. Which patients are at risk for developing a recurrence of reflex sympathetic dystrophy in the same or another limb. Pain 64:463-466

Veldman PH, Reynen HM, Arntz IE, Goris RJ (1993) Signs and symptoms of reflex sympathetic dystrophy: prospective study of 829 patients. Lancet 342:1012-1016

Watkins LR, Maier SF (2002) Beyond neurons: evidence that immune and glial cells contribute to pathological pain states. Physiol Rev 82:981-1011

Woda A, Pionchon P (2000) A unified concept of idiopathic orofacial pain: pathophysiologic features. J Orofacial Pain 14:196-212

Woolf CJ, Salter MW (2000) Neuronal plasticity: increasing the gain in pain. Science 288:1765-1769 